\title{
Dinamización de la clase magistral en medicina: diez ejemplos de minicasos utilizados en la docencia de la farmacología
}

\author{
J.E. Baños, M. Farré
}

Resumen. Las clases magistrales constituyen un método pedagógico muy utilizado en las facultades de medicina. Sin embargo, tiene diversas limitaciones entre las que se encuentran la pasividad y la pérdida de la atención de los alumnos. A fin de estimular la atención y revigorizar su empleo, se han empleado textos cortos que plantean preguntas que pueden resolverse con la información proporcionada en la clase magistral (minicasos). El presente artículo describe una experiencia de su uso en la docencia de la farmacología y ofrece diez ejemplos para ilustrar sobre su empleo. La mayoría de ellos están ambientados en el mundo real, permiten contextualizar los conocimientos básicos de farmacología y estimulan la comprensión de los conceptos claves.

Palabras clave. Aprendizaje activo. Clases magistrales. Farmacología. Minicasos.

Improving lectures in Medicine: ten examples of mini-cases to be used in the teaching of pharmacology

Summary. Lectures are the preferred method of teaching in many schools of Medicine. However, this method has several limitations, such as the passive role assumed by students and the loss of their attention. In order to improve the use of lectures, some authors have suggested the use of minicases. These are short texts that include several questions, which are related with the contents of the lecture and students are asked to answer them at its end. The present paper describes an experience of using minicases in the teaching of pharmacology. It offers ten examples to illustrate how they are built. Most of them are related with the real world, they permit to put knowledge of basic sciences in context of clinical problems and stimulate the understanding of key concepts of the discipline.

Key words. Active learning. Lectures. Minicases. Pharmacology.

\section{Introducción}

A pesar de las múltiples innovaciones realizadas en las últimas décadas en la docencia de la medicina, es razonable pensar que la clase magistral (o quizá mejor, la clase teórica) continuará siendo un recurso indispensable en los próximos años. Aceptando sus múltiples limitaciones, no cabe duda de que en muchos ámbitos seguirá constituyendo el principal medio de transmisión de la información. La tradición y la economía de recursos que precisa su empleo juega a su favor frente a otros métodos que precisan cambios sustanciales en la mentalidad de profesores y alumnos, así como una inversión en tiempo y personal que no todas las universidades pueden o desean realizar [1]. Por tanto, no es arriesgado afirmar que las clases magistrales, en mayor o menor número, permanecerán con nosotros largo tiempo.
Aceptada tal inevitabilidad, parece razonable introducir cambios en las clases teóricas que permitan amortiguar algunas de sus mayores limitaciones, como la pasividad de los alumnos o la pérdida de concentración a partir de los 15 minutos de iniciarse [2]. Además, no es infrecuente que lo que se explica carezca de una adecuada contextualización, lo que impide vislumbrar su utilidad real. Este segundo aspecto es especialmente relevante en las disciplinas básicas de los estudios de medicina [3]. Con la excepción de la anatomía, el resto de disciplinas pueden tener dificultades para trasladar a los estudiantes su importancia y la necesidad de aprenderlas más allá de los exámenes correspondientes, a pesar de que más tarde sean contempladas como muy importantes para la formación. La farmacología no es una excepción y es habitual que los estudiantes no comprendan su importancia hasta que
Departamento de Ciencias Experimentales y de la Salud; Universitat Pompeu Fabra (J.E. Baños). Departamento de Farmacología, de Terapéutica y de Toxicología; Universitat Autònoma de Barcelona (M. Farré). Institut Municipal d'Investigació Mèdica (IMIM-Hospital del Mar); Barcelona, España (M. Farré).

Correspondencia:

Dr. Josep Eladi Baños. Departamento de Ciencias Experimentales y de la Salud. Universitat Pompeu Fabra. Parc de Recerca Biomèdica de Barcelona. Doctor Aiguader, 88. E-08003 Barcelona.

E-mail:

Josepeladi.banos@upf.edu

C 2011 Educación Médica 
se encuentran a punto de dejar la facultad [4]. En los últimos años hemos intentado transmitirles que la disciplina es más que una asignatura a superar mediante la utilización de los minicasos, cuya experiencia piloto ya se publicó previamente en esta revista [5]. El procedimiento es siempre el mismo. Brevemente, antes del inicio de la clase magistral se entrega a los estudiantes una hoja con el texto del minicaso y las preguntas asociadas. Se lee conjuntamente y se procede a iniciar la clase magistral, que trata de conceptos que permiten entender el minicaso y contestar las preguntas planteadas. Al finalizar la clase, el profesor deja cinco minutos a los estudiantes para proceder a la respuesta escrita de las preguntas. Pasado este tiempo, se recogen los textos y se dan las respuestas correctas, con un comentario posterior si es oportuno. La idea no es estrictamente novedosa y existen ejemplos aplicados en otros ámbitos [6,7].

En el presente artículo presentamos los minicasos empleados en los últimos años en el área de la farmacología del sistema nervioso periférico y de los mediadores celulares, a fin de ilustrar esta posibilidad docente con varios ejemplos que puedan ser utilizados por los lectores en su ámbito docente. Algunos son una invención directa de los autores, mientras que otros se inspiran en situaciones reales y, cuando es así, se adjuntan referencias bibliográficas para ilustrarlos.

\section{Minicaso 1. Los nervios del estreno}

\section{Texto}

Joan se encuentra como un flan por los nervios que le crea el estreno de la obra de teatro que lleva ensayando desde hace meses. En ella interpreta el papel de maestro y tiene que explicar una lección a sus supuestos alumnos, por lo que debe escribir en la pizarra. Nota en su cuerpo una serie de sensaciones que no cree que lo ayuden demasiado en su debut en el escenario. ¿Podrá salir airoso de la experiencia?

\section{Preguntas y respuestas}

- ¿Qué respuesta neurovegetativa está experimentando? Joan está experimentando una activación de su sistema simpático a consecuencia del cuadro de ansiedad que le causa el estreno.

- ¿Qué consecuencias de esta respuesta pueden perjudicarle en el estreno? La activación simpática puede producirle una taquicardia (manifestada como palpitaciones) que aumente más su ansiedad, una activación muscular que le cause temblor de las extremidades y dificultades para realizar acciones precisas en escena, y sequedad de boca que alterará su capacidad de recitar su texto en la obra.

- ¿Qué fármaco elegiría para dicha sintomatología? En el contexto del minicaso, la mejor opción, siempre bajo prescripción médica, sería un antagonista de los adrenoceptores $\beta$ que atravesara la barrera hematoencefálica, como el propranolol.

- ¿Por qué no debería escoger un 'sedante?? Un 'sedante', es decir, un ansiolítico, podría perjudicarle por la probable producción de somnolencia, que podría alterar su estado de conciencia y afectar gravemente a su desempeño sobre el escenario.

\section{Comentario}

Este minicaso se utiliza para introducir los aspectos fisiológicos del sistema nervioso periférico que pueden tener importancia más adelante para comprender las posibles intervenciones farmacológicas que puedan realizarse $[8,9]$. Permite ilustrar con atención uno de los principales sistemas, el simpático, cuya activación se manifiesta claramente en situaciones de ansiedad como la que aflige al protagonista del minicaso. Asimismo, la segunda pregunta permite seleccionar aquellas manifestaciones de la actividad simpática que pueden perjudicarle en el caso de la actuación teatral, como palpitaciones, temblor, sudoración o sequedad de boca.

\section{Minicaso 2. iCuidado con la napia!}

\section{Texto}

Desde que se inicia la primavera, la nariz de Rosa no cesa de manar. Ello le impide rendir bien en su equipo de baloncesto, pues se pasa medio partido sonándose en el pañuelo. Como no tiene tiempo de ir al médico, toma prestado un nebulizador nasal que utiliza su hermana, que sufre el mismo problema y a la que alivia mucho. Después de unos días se encuentra bastante mejor, pero el problema ahora es que ha dado positivo en un control antidopaje. Rosa está segura de que no ha tomado nada que estuviera prohibido y cree que debe ser un error.

\section{Preguntas y respuestas}

- ¿Qué sustancia puede contener el nebulizador y por qué la alivia? Estos preparados llevan con frecuencia agonistas de los adrenoceptores $\alpha_{1} \mathrm{o}$ 
fármacos que facilitan la transmisión adrenérgica, cuyo efecto vasoconstrictor tiene utilidad terapéutica. Si está prohibido en deportistas, debe ser un compuesto con acciones sobre el sistema nervioso central. El más probable de los sospechosos es la efedrina.

- ¿Por qué razón está prohibido el uso de tales sustancias en deportistas? Los efectos estimulantes de la efedrina sobre el sistema nervioso central podían utilizarse en la práctica del dopaje, como en el pasado se emplearon las anfetaminas.

- ¿Qué podría utilizar como alternativa al nebulizador? Rosa podría utilizar un agonista de los adrenoceptores $\alpha_{1}$ que no atraviese la barrera hematoencefálica, ya fuera en forma de otro nebulizador (p. ej., oximetazolina o fenilefrina) o un preparado oral (p. ej., fenilefrina o pseudoefedrina).

\section{Comentario}

El minicaso 2 es especialmente útil cuando se explican los fármacos que activan el sistema simpático. Permite que los estudiantes comprendan que tales sustancias pueden tener efectos útiles en problemas de la vida común, pero con efectos indeseables desconocidos por los usuarios. En esta ocasión, el minicaso se ha centrado en fármacos que favorecen la transmisión adrenérgica, como los agonistas de los adrenoceptores $\alpha_{1}$, las anfetaminas o la efedrina $[10,11]$. Éstos ejercen efectos vasoconstrictores que son terapéuticos en la situación de Rosa. Sólo los dos últimos tienen marcados efectos estimulantes sobre el sistema nervioso central. Dada la prohibición del uso de estimulantes de tipo anfetaminas en deportistas, la sustancia incriminada es claramente la efedrina u otras de tipo anfetamínico que podrían estar presentes en el nebulizador [12]. Debería utilizar otros fármacos agonistas de los adrenoceptores $\alpha_{1}$, sin acción central y que pueden administrarse tanto por vía nasal como por vía oral. Además, los vasoconstrictores pueden causar efectos de rebote tras su administración repetida que desaconsejan su empleo de forma continuada.

\section{Minicaso 3. Barça-Madrid}

\section{Texto}

Pere es socio del Barça desde que nació. Es culé hasta la médula y sufre lo indecible con los partidos de su equipo. Hoy está en el campo viendo el encuentro contra su máximo rival y el desenlace es incierto. Durante la primera parte del partido nota los ner- vios a flor de piel y siente que su corazón late rápidamente y con mucha fuerza. Al llegar el descanso comenta a su amigo Sergi que nota palpitaciones. Éste le contesta que a él también le sucedía, pero que toma unos comprimidos que le quitan esa sensación. Pere le pide uno, pero Sergi le pregunta si es asmático. Cuando lo niega, Sergi se lo entrega. El comprimido obra efectos mágicos: durante la segunda parte, las palpitaciones desaparecen y el pulso de Pere no supera los sesenta latidos por minuto.

\section{Preguntas y respuestas}

- ¿Qué fármaco puede contener el comprimido? Con toda probabilidad, un antagonista de los adrenoceptores $\beta$ como el propranolol, dada la reducción marcada de la frecuencia cardíaca.

- ¿Por qué Sergi le preguntó si era asmático? Un efecto indeseable de los antagonistas no selectivos de los adrenoceptores $\beta$ es el desencadenamiento de un episodio de broncoespasmo en las personas que sufren asma bronquial, por lo que se desaconseja su administración en tales pacientes.

\section{Comentario}

El minicaso 3 ilustra el uso de los antagonistas de los adrenoceptores $\beta$ en una indicación terapéutica que no es la principal, pero que permite comprender claramente su mecanismo de acción [11,13]. Además, la referencia a la posibilidad de la enfermedad asmática del protagonista ayuda a introducir el problema de los efectos indeseables y la multiplicidad de efectos fisiológicos de los medicamentos. Adicionalmente, ayuda a diferenciar unos antagonistas de otros, pues no todos son igual de selectivos sobre los subtipos de receptores. Está inspirado en un ejemplo de Laurence y Bennett [14].

\section{Minicaso 4. Los peligros de los invernaderos}

\section{Texto}

En una plantación de flores del Maresme, Dawda Jawara acaba de iniciar su jornada laboral. Recién llegado de Gambia, le han encargado que atienda las rosas que se están desarrollando en el invernadero. Cuando entra, se cruza con unos compañeros que van a recoger unas herramientas. Le sorprende encontrar una neblina extraña, pero no hace mucho caso y empieza a trabajar. Al regresar sus compañeros un par de horas después, lo encuentran inconsciente y con saliva que se escapa por sus comisuras labiales. 
Preguntas y respuestas

- ¿Qué le puede haber sucedido a Dawda desde que sus compañeros lo vieron por última vez? Es muy probable que se haya intoxicado con los plaguicidas que se utilizan en las plantaciones para evitar el ataque de los parásitos. En nuestro ámbito es frecuente el empleo de organofosforados para alcanzar este objetivo.

- ¿Qué signos son más probables al realizar la exploración física básica? Los signos más probables que se encontrarán en el afectado son la presencia de intensa miosis, congestión ocular, bradicardia, fasciculaciones musculares, incontinencia urinaria, diarrea, sudoración e hipotensión, entre otros.

\section{Comentario}

El minicaso 4 introduce la farmacología desde su vertiente más toxicológica y pretende que los estudiantes relacionen el efecto de un fármaco con un cuadro clínico específico. Los organofosforados son un buen ejemplo para explicar cómo pueden afectarse las sinapsis colinérgicas y los resultados de una activación excesiva $[15,16]$.

En la segunda pregunta se pretende establecer el significado clínico de tal alteración fisiológica, lo que debería permitir la comprensión y la contextualización de un fenómeno probablemente demasiado abstracto para los estudiantes de tercer curso como es la inhibición de la acetilcolinesterasa. Contribuye además a relacionar la farmacología con la semiología básica de la intoxicación.

\section{Minicaso 5. Las sustancias naturales nunca hacen daño}

\section{Texto}

Hospital del Mar, servicio de urgencias, madrugada del domingo. Un chico de veinte años entra acompañado de varios amigos. Dice cosas raras, parece no reconocer a sus compañeros y se niega a obedecer las peticiones de los profesionales sanitarios. Cuando consiguen acostarlo en la camilla, se dan cuenta de que tiene la piel seca y caliente. La exploración revela una manifiesta taquicardia, sin otras alteraciones de interés. Sus amigos juran que no ha tomado alcohol ni ninguna droga. 'Es imposible -afirman-, Xavier es muy estricto y sólo toma infusiones de hierbas'.

\section{Preguntas y respuestas}

- ¿Qué le está pasando a Xavier desde el punto de vista neurovegetativo y qué sencilla maniobra de exploración contribuiría al diagnóstico? Las manifestaciones son de una activación simpática (taquicardia) o de una más probable inhibición parasimpática (piel seca y caliente). La exploración de la pupila ayudaría al diagnóstico.

- ¿Podría existir alguna relación entre su afición por las infusiones vegetales y el cuadro clínico que presenta? Podría. Su semiología clínica es compatible con una intoxicación anticolinérgica, por lo que dada su afición a la botánica podría relacionarse con la toma de infusiones de preparados vegetales de beleño, mandrágora o belladona.

\section{Comentario}

El minicaso 5 describe un nuevo cuadro de intoxicación, en este caso por plantas que contienen antimuscarínicos, algo no infrecuente en los servicios de urgencias [17]. Con el texto se desea que los estudiantes comprendan lo que puede ocurrir cuando se toman alcaloides naturales que tienen una elevada capacidad para causar manifestaciones clínicas singulares $[18,19]$. También se desea que se reconozca la importancia del conocimiento de los efectos farmacológicos para escoger exploraciones físicas que puedan desvelar signos de gran importancia.

\section{Minicaso 6. Un caso para el sargento Sabihondo}

\section{Texto}

Mossos d'Esquadra. División de la Policía Científica. Un grupo de delincuentes de un país de la antigua Unión Soviética está actuando en Cataluña, cuya especialidad es el secuestro. Inmovilizan a las víctimas con una inyección intramuscular de sustancias desconocidas que producen una rápida parálisis muscular, con lo que son incapaces de defenderse. El informe de Interpol establece que el efecto de tales sustancias puede revertirse por un antídoto, pero que éste no siempre funciona. Todo es muy misterioso, pero el sargento Sabihondo tiene la respuesta.

\section{Preguntas y respuestas}

- ¿Qué sustancias podrían utilizar los delincuentes y cómo actúan? Los datos que ofrece el texto 
inducen a pensar en el empleo de paralizantes musculares que actuarían sobre la placa motora. Podrían bloquear la activación muscular ya sea actuando sobre el receptor nicotínico (bloqueantes no despolarizantes, como el pancuronio o el atracurio) o despolarizándolo por su activación repetida sobre él (bloqueantes despolarizantes, como la succinilcolina).

- ¿Cuál sería el antídoto y por qué a veces no es efi$c a z$ ? El antídoto sería la administración de un anticolinesterásico (neostigmina) junto con atropina. $\mathrm{Su}$ eficacia dependería de cuál fuera la sustancia empleada, ya que el anticolinesterásico es ineficaz para revertir la parálisis muscular causada por la administración de bloqueantes despolarizantes.

\section{Comentario}

Este minicaso tiene el objetivo de destacar el interés de conocer el funcionamiento de la placa motora, de las sustancias que pueden facilitar la transmisión y de las que pueden bloquearla [20,21]. Obviamente, el minicaso no tiene una respuesta única en ninguna de sus preguntas y se pide que los estudiantes realicen una aproximación reflexiva y razonada a su solución. Muestra, además, que incluso los fármacos más especializados pueden utilizarse fuera del ámbito médico con objetivos delictivos. No se conoce el empleo de tales sustancias con esa intención en el mundo real, pero más de un autor las ha utilizado en la ficción con fines homicidas [22,23].

\section{Minicaso 7. Zombis farmacológicos}

Texto

Los zombis haitianos o 'muertos vivientes' se encuentran entre la fantasía y la realidad. Mónica ha leído que los brujos caribeños son capaces de crearlos. Para ello, envenenan la comida de las víctimas con una sustancia natural que les produce una parálisis nerviosa, lo que las deja en un estado de muerte aparente. Después las entierran y, cuando todo el mundo cree que han fallecido, las sacan de la tumba y les hacen creer que les han devuelto la vida y que pertenecen a los brujos para siempre. Mónica se pregunta si toda esta historia es verdad, pero no puede evitar que un escalofrío le recorra la espalda.

\section{Preguntas y respuestas}

- ¿Qué tipo de sustancias podrían producir los efectos descritos? Las sustancias naturales que pue- den provocar tales efectos son principalmente las toxinas que actúan sobre los canales de sodio, en especial las que bloquean su apertura.

- ¿Sobre qué tejidos actuarían para provocar el estado de muerte aparente y cuál es la sustancia natural que podría producirlo con más probabilidad? Actuarían sobre tejidos excitables (nervioso, cardíaco) de forma que reducirían notablemente la probabilidad de su activación por su dependencia de los canales de sodio para ello. Las más probables serían la tetrodotoxina y la saxitonina, por su frecuente presencia en organismos marinos.

\section{Comentario}

Este minicaso se basa en una curiosa historia, que tuvo cierta resonancia hace algunos años, sobre la posibilidad de que los zombis fueran en realidad personas intoxicadas intencionadamente con tetrodotoxina [24-26]. Fuera cierta o no (más bien no), es un buen argumento para analizar lo que puede ocurrir cuando se bloquean los canales de sodio con toxinas que se absorben por vía oral [27].

\section{Minicaso 8. Malditos picores}

\section{Texto}

Mario sufría una intensa urticaria a consecuencia de una alergia de la que aún desconocía la causa. Al entrar en la cabina del avión pidió un vaso de agua y se tomó una cápsula para aliviarse del prurito insoportable. El viaje a Ibiza tenía pocas dificultades, buen tiempo y excelente visibilidad. El despegue fue bien, pero poco después comenzó a adormilarse y los ojos se le cerraban sin que pudiera evitarlo. Suerte del piloto automático, pero en el momento de aterrizar la cosa no fue bien. Se dispararon las alarmas de salida de pista: iiiplane crash!!! Suerte que todo ocurrió en el simulador de vuelo de la escuela de aviación de Reus. Cuando consultó con el instructor de vuelo, descubrió que los pilotos tenían prohibidos muchos medicamentos y que para las alergias sólo podían tomar los fármacos que recomendaba la Federal Aviation Administration (FAA) estadounidense.

\section{Preguntas y respuestas}

- ¿Qué tipo de sustancia había tomado Mario? El prurito asociado a la urticaria responde bien a la administración de antihistamínicos, ya que la histamina suele estar implicada con frecuencia 
en fenómenos alérgicos. Dada la somnolencia que apareció lo más probable es que se tratara de un antihistamínico $\mathrm{H}_{1}$ de primera generación (p. ej., clorfeniramina).

- ¿Qué fármacos pudo recomendarle el instructor de vuelo para las alergias? En caso de contraindicación absoluta a los efectos sedantes de los antihistamínicos, se debería utilizar un antihistamínico $\mathrm{H}_{1}$ de segunda generación, que carece de tales efectos (p. ej., loratadina).

\section{Comentario}

Este minicaso se dirige a ilustrar la capacidad de algunos antihistamínicos para producir un efecto indeseable frecuente $y$ potencialmente peligroso $[28,29]$. Además, pretende que los estudiantes comprendan el interés de la selectividad farmacológica para evitar algunas reacciones adversas. También se pretende que los estudiantes comprendan cómo el desarrollo farmacológico puede contribuir a mejorar fármacos con buena eficacia pero con un perfil bajo de seguridad. En la página web de la FAA se encuentra la lista de medicamentos autorizados y prohibidos [30].

\section{Minicaso 9. El último tango}

\section{Texto}

Quimeta tenía 67 años y hacía unos meses que le habían diagnosticado una insuficiencia cardíaca. La situación estaba bajo control, e incluso había vuelto a bailar tangos y pasodobles cada sábado en su club de baile de la tercera edad. Hace unos días empezó a padecer un dolorcillo en las rodillas que se le pasaba tomando antiinflamatorios durante unos días y con un poco de paciencia... Empezó a tomar las pastillas contra el dolor. Un par de días después notó que las piernas se le hinchaban y le costaba un poco respirar. 'No será nada - pensó-, debo ir al concurso de baile del sábado'. El día que se celebró éste, tenía las piernas muy hinchadas y le costó llegar hasta la sala de baile. No se pudo poner los zapatos de baile y le costaba respirar. Al sonar el primer tango se levantó y con el primer movimiento ya notó la falta de aire y el dolor en el pecho. ‘¿Dios mío!, a ver si este será mi último tango'.

\section{Preguntas y respuestas}

- ¿Qué puede estar pasándole a Quimeta? La administración de antiinflamatorios no esteroideos
(AINE) puede producir retención hidrosalina. En el caso de Quimeta, es probable la descompensación de la insuficiencia cardíaca, que se manifiesta en la aparición de edemas en las extremidades inferiores (piernas hinchadas) y un principio de edema pulmonar (falta de aire y dolor en el pecho).

- ¿Qué fármacos pueden ser los responsables del empeoramiento de su enfermedad y mediante qué mecanismo podrían estar actuando? Los fármacos que la producen son muy probablemente los AINE que causarían la descompensación, cuando la inhibición de la síntesis de prostaglandinas renales supone una retención de sodio y agua, un aumento del volumen intravascular y el consiguiente agravamiento de la insuficiencia cardíaca.

\section{Comentario}

Este minicaso constituye un ejemplo de la integración de una situación clínica con elementos básicos de la farmacología que, en ocasiones, pueden ser difíciles de relacionar (y de valorar) por los estudiantes. La situación descrita permite comprender además cómo un efecto indeseable de tipo colateral puede tener importancia clínica en personas predispuestas [31].

\section{Minicaso 10. El insoportable dolor mensual}

Texto

Desde que llegó por primera vez, su cita mensual fue una tortura para Consuelo. Los espasmos en la pelvis, el dolor en la zona lumbar, el malestar y el mal humor eran una tortura que debía soportar cada cuatro semanas. Afortunadamente, una visita a la ginecóloga pudo librarla de semejante tormento. Tras explicarle por qué se producía, le prescribió un fármaco que le ha aliviado notablemente de todas sus manifestaciones dolorosas. Se pregunta cómo no lo descubrió antes y qué tiene de mágica esta sustancia.

\section{Preguntas y respuestas}

- ¿Qué fármaco le prescribió la ginecóloga y cómo actúa para ejercer su efecto analgésico? Muy probablemente le prescribió un AINE, ya que este tipo de fármacos son muy eficaces para reducir el dolor menstrual por la inhibición de la síntesis de prostaglandinas en el útero y por sus efectos 
analgésicos adicionales en el sistema nervioso central.

- ¿Qué mediador celular es el responsable del dolor de Consuelo y cómo lo produce? La prostaglandina $\mathrm{F}_{2 \alpha}$ es la principal responsable del dolor menstrual por sus efectos sensibilizantes de los nociceptores periféricos y por la contracción de las fibras musculares del miometrio.

\section{Comentario}

El complejo mundo de los mediadores celulares y de la inflamación puede ser de difícil comprensión para los estudiantes de medicina sin una adecuada contextualización. En este minicaso se intenta que se conozca la asociación de uno de ellos con una situación común, la dismenorrea. Mediante la disección fisiopatológica de sus acciones, los estudiantes pueden comprender cómo intervenir farmacológicamente en un mecanismo dado con un buen éxito terapéutico $[32,33]$.

\section{Conclusiones}

En los últimos años se han realizado numerosos intentos de mejorar la docencia de la medicina, en sus aspectos filosóficos, teóricos, prácticos y docimológicos. En la mayoría de los casos, tales mejoras pasan por la utilización de métodos de aprendizaje colaborativo que, en general, precisan de grupos pequeños [34]. Sin embargo, no se ha prestado tanta atención a la posibilidad de intervenir en el método docente por excelencia en nuestro ámbito, la clase magistral. Debido a diversas razones, como las económicas, las logísticas o las psicológicas, es difícil pensar que este método desaparecerá de nuestras aulas en un futuro próximo. Por ello, parece razonable buscar alternativas que, salvaguardando su estructura y el grupo clase, permitan dinamizarla de manera que rebase el límite tradicional de la transmisión pasiva de información. En esta dirección proponemos que el empleo de minicasos, en conjunción con la clase magistral, puede ayudar a dar un nuevo interés a un recurso docente de contrastada eficacia, pero que se atenúa cuando se abusa de él sin transmitir su interés a los estudiantes.

Los minicasos tienen indudables ventajas. Permiten una concepción adaptada a las necesidades docentes de cada grupo, son fáciles de elaborar, permiten su utilización en grupos clase, son de fácil evaluación y pueden contribuir a aumentar la asistencia y la motivación de las clases magistrales [5]. Este hecho no es baladí, si se tiene en cuenta que la presencia de los estudiantes en las actividades presenciales contribuye a mejorar el rendimiento académicos [35]. En las asignaturas básicas contribuyen además a facilitar la contextualización del conocimiento y permiten que los estudiantes comprendan de forma sincrónica la importancia de la disciplina [6,7]. En el presente artículo presentamos diversos ejemplos aplicados a una parte de la farmacología que puede ser difícil de comprender por los estudiantes y más aún de apreciar su interés para las disciplinas que cursarán a continuación. Como ya se ha descrito [5], la aceptación por los estudiantes es buena y constituye en la actualidad uno de los elementos docentes que aplicamos de forma rutinaria desde hace varios años.

Algunos profesores pueden ver ciertas dificultades en su empleo. Por ejemplo, no realizamos un control de que el resultado del trabajo sea individual, ya que los estudiantes trabajan consultándose unos a otros. Ello puede considerarse una limitación porque la evaluación no recoge el esfuerzo de cada estudiante. Esta consideración es obviamente cierta, pero creemos que el intercambio de información y de opiniones constituye también una forma de aprender en grupo que no es limitante siempre que se disponga de otros medios de evaluación. Otro aspecto es el problema clásico de la transmisión de los minicasos de un curso a otro de forma que se puedan 'copiar' los resultados. No descartamos esta posibilidad, pero creemos que tiene una incidencia menor en la mayoría de las clases porque, en nuestra experiencia, la respuesta a las preguntas viene precedida en la mayoría de los casos de numerosas consultas a los apuntes de clase y a los compañeros. Ello nos sugiere que no es un problema importante. En tercer lugar, algunos profesores pueden preferir aprovechar todo el tiempo disponible de clase para la exposición en lugar de limitarlo para permitir la realización de los minicasos. Nuestra opinión es contraria a forzar al máximo el tiempo de clase para la transmisión de conocimientos. De hecho, el Espacio Europeo de Educación Superior aboga por un mayor trabajo autónomo del alumno, lo que debería cumplirse fuera del aula y, por tanto, el rol del profesor no debería ser el de un proveedor exhaustivo de conocimiento [36]. En esta creencia, la reducción de unos minutos no debería afectar su capacidad docente.

En definitiva, creemos que los minicasos son un elemento didáctico de gran interés, con una relación inversión-beneficio elevada y una notable capacidad para mejorar la asistencia a clase y la comprensión de las asignaturas. En el presente artículo se han expuesto diversos ejemplos que pueden uti- 
lizarse con este fin en la docencia de la farmacología en medicina u otras disciplinas biomédicas. Sin embargo, la idea es de aplicación posible a la mayoría de las materias que se imparten en las facultades universitarias, previa adaptación a la idiosincrasia de cada asignatura y cada titulación.

Bibliografía

1. Hatton N. ¿Cómo diseñar e impartir una clase? In Cox KR, Ewan CE, eds. La docencia de la medicina. Barcelona: Doyma; 1990. p. 34-42.

2. Stuart J, Rutherford RJD. Medical student concentration during lectures. Lancet 1978; 2: 514-6.

3. Flaquer A. Punto de vista del estudiante de medicina sobre la formación de pregrado. Educ Med 2001; 4: 111-2.

4. De Juan J, Martínez-Cruz F, Cuenca N, Fernández E, GarcíaBarbero M. Importancia de las asignaturas preclínicas en la formación del médico. Rev Clin Esp 1988; 183: 42-7.

5. Farré M, Baños JE. Los minicasos como un método docente adyuvante a las clases magistrales: la opinión de los estudiantes sobre una prueba piloto en farmacología. Educ Med 2006; 9: 134-7.

6. Lubawy WC, Brandt BF. A variable structure, less resource intensive modification of problem-based learning for pharmacology instruction to health science students. Naunyn Schmiedeberg's Arch Pharmacol 2002; 366: 48-57.

7. Lubawy WC, Brandt BF. Microsituations as an active-learning tool to teach endocrine pharmacology and problem-solving skills. Am J Pharm Educ 1998; 62: 333-41.

8. Westfall TC, Westfall DP. Neurotransmisión. Sistemas nerviosos autónomo y motor somático. In Brunton LL, Lazo JS, Parker KL, eds. Goodman \& Gilman. Las bases farmacológicas de la terapéutica. 11 ed. México DF: McGraw-Hill; 2007. p. 137-81.

9. Flórez J. Farmacología general del sistema nervioso autónomo. In Flórez J, ed. Farmacología humana. 5 ed. Barcelona: Elsevier Masson; 2008. p. 261-7.

10. García-Sevilla JA, Meana JJ. Transmisión catecolaminérgica. Fármacos agonistas catecolaminérgicos. In Flórez J, ed. Farmacología humana. 5 ed. Barcelona: Elsevier Masson; 2008. p. 295-319.

11. Westfall TC, Westfall DP. Agonistas y antagonistas adrenérgicos. In Brunton LL, Lazo JS, Parker KL, eds. Goodman \& Gilman. Las bases farmacológicas de la terapéutica. 11 ed. México DF: McGraw-Hill; 2007. p. 237-97.

12. Resolución de 18 de diciembre de 2009 , de la Presidencia del Consejo Superior de Deportes, por la que se aprueba la lista de sustancias y métodos prohibidos en el deporte. BOE del 25 de diciembre de 2009; p. 109755-60.

13. Meana JJ, García-Sevilla JA. Fármacos que modifican la actividad simpática. In Flórez J, ed. Farmacología humana. 5 ed. Barcelona: Elsevier Masson; 2008. p. 321-35.

14. Laurence DR, Bennett PN. Clinical pharmacology. 5 ed. London: Churchill-Livingstone; 1980.

15. González-García C, Martín ED, Fernández M. Transmisión colinérgica. Fármacos agonistas colinérgicos. In Flórez J, ed. Farmacología humana. 5 ed. Barcelona: Elsevier Masson; 2008. p. 269-85.
16. Taylor P. Anticolinesterasa. In Brunton LL, Lazo JS, Parker KL, eds. Goodman \& Gilman. Las bases farmacológicas de la terapéutica. 11 ed. México DF: McGraw-Hill; 2007. p. 201-15.

17. Jiménez-Mejías ME, Fernández A, Montaño-Díaz M, González de la Puente MA. Síndrome anticolinérgico por envenenamiento por Datura stramonium. Med Clin 1991; 97: 237-8.

18. Heller Brown J, Taylor P. Agonistas y antagonistas de los receptores muscarínicos. In Brunton LL, Lazo JS, Parker KL, eds. Goodman \& Gilman. Las bases farmacológicas de la terapéutica. 11 ed. México DF: McGraw-Hill; 2007. p. 183-99.

19. Ceña V, Posadas I. Fármacos antagonistas colinérgicos muscarínicos. In Flórez J, ed. Farmacología humana. 5 ed. Barcelona: Elsevier Masson; 2008. p. 287-93.

20. Badía A, Baños JE. Fármacos bloqueantes de la placa motriz y bloqueantes ganglionares. In Flórez J, ed. Farmacología humana. 5 ed. Barcelona: Elsevier Masson; 2008. p. 337-53.

21. Taylor P. Agentes que actúan en la unión neuromuscular y en los ganglios autónomos. In Brunton LL, Lazo JS, Parker KL, eds. Goodman \& Gilman. Las bases farmacológicas de la terapéutica. 11 ed. México DF: McGraw-Hill; 2007. p. 217-35.

22. Christie A. Death in the air. New York: Dodd Mead; 1935.

23. Cleary J. Dark summer. New York: Harper \& Collins; 1992.

24. Puffers, gourmands and zombification [editorial]. Lancet 1984; 323: 1220-1.

25. Davis EW. The ethnobiology of the Haitian zombi. J Ethnopharmacol 1983; 9: 85-104.

26. Booth W. Vodoo science. Science 1988; 240: 274-7.

27. Rang HP, Dale MM, Ritter JM, Flower RJ. Rang y Dale. Farmacología. 6 ed. Barcelona: Elsevier Churchill Livingstone; 2008.

28. Pazos A. Mediadores celulares I. Histamina y 5-hidroxitriptamina Farmacología de la migraña. In Flórez J, ed. Farmacología humana. 5 ed. Barcelona: Elsevier Masson; 2008. p. 367-89.

29. Skidgel RA, Erdös EG. Histamina, bradicinina y sus antagonistas. In Brunton LL, Lazo JS, Parker KL, eds. Goodman \& Gilman. Las bases farmacológicas de la terapéutica. 11 ed. México DF: McGraw-Hill; 2007. p. 629-51.

30. Federal Aviation Administration. Pilot medical solutions. FAA accepted medications. Updated 4/09/09. URL. http:// www.leftseat.com/medcat1.htm. [04.02.2010].

31. Feria M. Fármacos analgésicos antitérmicos y antiinflamatorios no esteroideos. Antiartríticos. In Flórez J, ed. Farmacología humana. 5 ed. Barcelona: Elsevier Masson; 2008. p. 421-55.

32. Esplugues JV, Barrachina MD. Mediadores celulares II. Eicosanoides, óxido nítrico y factor activador de plaquetas. In Flórez J, ed. Farmacología humana. 5 ed. Barcelona: Elsevier Masson; 2008. p. 391-405.

33. Burke A, Smyth E, Fitzgerald GA. Autacoides derivados de lípidos: eicosanoides y factor activador plaquetario. In Brunton LL, Lazo JS, Parker KL, eds. Goodman \& Gilman. Las bases farmacológicas de la terapéutica. 11 ed. México DF: McGraw-Hill; 2007. p. 653-69.

34. Amat O. Aprender a enseñar. 6 ed. Barcelona: Profit; 2009.

35. Pérez J, Graell S. Asistencia a clase y rendimiento académico en estudiantes de medicina. La experiencia de la Universidad Autónoma de Barcelona. Educ Med 2004; 7: 85-9.

36. Benito A, Cruz A, eds. Nuevas claves para la docencia universitaria en el Espacio Europeo de Educación Superior. Madrid: Narcea; 2005. 